\title{
Les nouvelles formes de la violence urbaine aux
} Etats-Unis

Sophie Body-Gendrot

\section{OpenEdition}

1 Journals

\section{Édition électronique}

URL : http://journals.openedition.org/conflits/647

DOI : $10.4000 /$ conflits. 647

ISSN : $1777-5345$

Éditeur :

CCLS - Centre d'études sur les conflits lilberté et sécurité, L'Harmattan

\section{Édition imprimée}

Date de publication : 16 juillet 1992

ISSN : 1157-996X

\section{Référence électronique}

Sophie Body-Gendrot, "Les nouvelles formes de la violence urbaine aux Etats-Unis », Cultures \&

Conflits [En ligne], 06 | été 1992, mis en ligne le 06 janvier 2003, consulté le 30 mars 2021. URL : http:// journals.openedition.org/conflits/647 ; DOI : https://doi.org/10.4000/conflits.647

Ce document a été généré automatiquement le 30 mars 2021.

Creative Commons License 


\title{
Les nouvelles formes de la violence urbaine aux Etats-Unis
}

\author{
Sophie Body-Gendrot
}

Les nouvelles formes de la violence urbaine aux Etats-Unis

Sophie BODY GENDROT ${ }^{1}$

3 Les émeutes qui ont éclaté à Los Angeles et dans une vingtaine de villes en mai 1992 confirment la représentation entretenue par les médias, le cinéma et la littérature : les Etats-Unis sont un pays d'une extrême violence. Les débordements associés au paysage urbain suggèrent que la violence est autant le fait des institutions légitimes qui les amplifient que des masses révoltées qui en sont à l'origine. Il s'agit en effet tout autant de la tyrannie de majorités et d'institutions (municipalités se désintéressant du sort de minorités victimes de brutalités ; jurys, forces de police violant les règles de la justice la plus élémentaire) que de révoltes de la part des opprimés. Pourtant, compte tenu de la charge explosive que représente cette société multiraciale et multiethnique, mal intégrée par une histoire relativement courte, par des institutions aux faibles pouvoirs et par une idéologie fortement individualiste, le nombre de conflits violents est relativement peu élevé comparé à la moyenne des conflits civils établie pour 124 nations et colonies ${ }^{2}$. Et si l'on compare la violence urbaine américaine à celle des villes de l'Amérique latine, et du Brésil en particulier, ou si l'on englobe dans la problématique la violence ethnique armée, celle de l'Irlande du Nord, de la Yougoslavie, du Liban et de certaines parties de la CEI, celle-ci apparaît relativement sporadique et limitée géographiquement. Notre propos consiste donc à montrer pourquoi les Etats-Unis, à l'exception de deux périodes : la Révolution et la Guerre civile, n'ont pas connu le sort des pays cités ; puis de distinguer toutefois les nouveaux facteurs qui font de la violence urbaine une réalité quotidienne, distinguant les EtatsUnis des autres nations développées tant par la nature que par l'ampleur de ce phénomène. Le durcissement des cassures sociales laisse augurer un avenir relativement sombre pour une majorité d'Américains.

Une violence instrumentale contenue 
4 Si par la technique d'un film projeté en accéléré, on déroule trois cents ans d'histoire américaine, la brutalité de la mentalité puritaine, du capitalisme et des clivages raciaux et sociaux caractérise les épisodes les plus saillants de cette histoire. Les colons puis les Américains se sont livrés à la violence pour exterminer les premiers occupants, punir les sorcières et les femmes adultères, lyncher les Noirs, mettre au pas les immigrants, incarcérer les Japonais accusés de trahison envers les Etats-Unis, défendre avec âpreté la conformité de leur espace territorial au plus petit échelon et selon la définition la plus étroite. La transplantation dans un Nouveau Monde et la levée des codes et inhibitions en usage en Angleterre ont suscité une extrême violence à l'égard des Amérindiens et des esclaves noirs, la Constitution ne reconnaissant dans les Noirs que les trois cinquièmes d'une personne et refusant d'accorder la citoyenneté aux ressortissants des tribus indiennes a légitimé les pratiques de violence à leur égard ; plus tard, d'inévitables heurts se sont produits au cours du XIXème siècle entre immigrants et Américains de souche, entre ouvriers et patrons pendant l'âge de la violence industrielle (Haymarket à Chicago en 1886; Homestead en Pennsylvanie et Coeur d'Alènes dans l'Idaho en 1892 ; Chicago encore et l'ouest de la région en 1894 et en 1904-05; Lawrence dans le Massachusetts en 1912 ; le pays tout entier en 1917-18 et 1919, année sans équivalent dans l'histoire ouvrière américaine) ; l'hystérie collective a pris forme légale à l'encontre des immigrants irlandais au milieu du XIXème siècle, des Asiatiques au tournant du XXème siècle, des Allemands et des esprits subversifs lors de la Première Guerre mondiale et au cours des années vingt surnommées à juste titre " les années tribales ", sans compter les meurtres de présidents et autres personnalités en vue ${ }^{3}$.

5 Ces quelques exemples historiques tendraient à suggérer que la violence fait partie intégrale de la formation de la nation américaine et de sa culture. Histoire d'une conquête faite à coups de revolvers et de sanglantes luttes ethniques. Dans certains cas, l'immensité de l'espace offert a permis de la contourner ou de la fuir (on pense à Roger Williams, puis aux Ammish) et l'espace a été protecteur d'idées, de cultures et de modes de vie différents ${ }^{4}$. Cette violence, en quelque sorte fondatrice, et liée à l'essor de la ville et à la diffusion du darwinisme social dans les rapports entre groupes et individus, s'est atténuée entre 1930 et $1955^{5}$; comme si après le recours des déracinés à des modèles traditionnels de violence rurale dans un environnement urbain, l'installation en ville avait fini par produire une génération plus docile, mieux socialisée, plus civilisée que les précédentes. La fermeture des portes aux immigrants, la Dépression et surtout un mode d'urbanisation favorisant les enclaves ont contribué à apaiser les conflits, le problème racial mis à part. C'est à cette époque que les Blancs se sont éloignés des Noirs, les riches des pauvres, les ouvriers les plus qualifiés et les mieux payés de leurs camarades moins fortunés. Selon S. Bass Warner, le double triomphe de l'entreprise industrielle moderne et de la banlieue du XXème siècle a créé des systèmes d'intégration et de ségrégation spatiale et sociale qui ont contribué à résoudre pendant cette période les conflits de classe, d'ethnie et de race (par exemple, le taux d'arrestation pour homicide à Boston en 1860 était de 7 pour 100 000, il était inférieur à 2 en 1950) ${ }^{6}$. Le pluralisme centriste, " nordiste " encouragé par des philosophes tels que William James, John Dewey et Horace Kallen a également favorisé l'essor d'expressions individuelles et collectives qui ont été intégrées avec le temps par le noyau dur anglosaxon devenu plus tolérant. La prospérité économique des années 1945-65 a également contribué à réduire les niveaux de tension. 
Pourtant, un second modèle antinomique du précédent caractérise les relations interraciales américaines et nourrit la violence : le modèle de caste, rigide, " sudiste " que résume ce court dialogue extrait des Aventures de Hukleburry Finn de Mark OTWain (publié en 1884) :

- Mon Dieu ! Quelqu'un s'est-il fait mal?

8

- Non, m'dme. Un Nègre tué.

- Eh bien, on a eu de la chance. Car quelquefois, les gens se font mal.

10

Outre les lois Jim Crow en vigueur dans le Sud (où en 1900 résident neuf Noirs sur dix) et les méfaits du Ku Klux Klan et d'autres bandes de citoyens armées, la violence raciale - soulèvement des minorités face à des provocations racistes et répression brutale n'épargne pas le Nord. Après avoir écrit que la maturation et la modernisation produisaient des villes paisibles, R. Lane a cherché à comprendre pourquoi tant de violence s'était exprimée au cours des années 1960 et 1970. Il s'est intéressé à la violence chez les Afro-Américains de Philadelphie à la fin du XIXème siècle et il a découvert que leur histoire ne correspondait pas à sa précédente théorie d'apaisement social. La confrontation raciale était déjà présente à Philadelphie avant la révolution américaine. En 1834, des affrontements brutaux opposaient immigrants irlandais et population noire (en 1863 à New York, entre 200 et 500 Noirs devaient être lynchés ou brûlés vifs par les immigrants irlandais refusant la conscription). Quelle que soit la durée de leur implantation dans la ville, les Afro-Américains de Philadelphie restaient plus liés à la criminalité que les composantes blanches de la population ${ }^{7}$. Ces dernières étaient intégrées dans la ville par les associations, les syndicats, les quartiers homogènes, les appareils politiques, tandis que les Afro-Américains étaient mis à l'écart par la société qui les avait produits et les avait laissés pourrir dans des ghettos assignés. Il n'est donc guère étonnant qu'ils aient pris les armes pour se venger et imposer un changement à leur situation d'infériorité institutionnalisée et que celui-ci tardant à venir, ils aient recommencé collectivement dans les années 1960. Cela dit, il est difficile d'établir un lien direct entre le niveau de tension sociale et l'éruption de la violence qui se produit plutôt sous l'effet d'un relâchement du contrôle imposé à la population opprimée. Ce serait abonder dans l'image des " classes dangereuses " promptes à la violence. Or s'il n'existe pas de causalités ni de récurrences scientifiquement démontrées dans ce domaine, le bon sens populaire a entériné l'idée probable selon laquelle l'occasion fait le larron. M. Janowitz distingue les émeutes " communales " précédant la Seconde Guerre mondiale des émeutes " matérialistes " (commodity) ${ }^{8}$. Les émeutes de Chicago en 1917 et 1919 ont trait à des définitions de frontières territoriales tout comme celles de Miami et de St Louis. Elles sont généralement précédées par le plastiquage des lieux d'habitation des Afro-Américains, signal que leur territoire ne doit pas empiéter sur celui des Blancs. Les deux premières phases du schéma écologique de l'Ecole de Chicago s'appliquent ici et vise à maintenir la ségrégation territoriale entre les races. A Harlem en 1943, un autre type d'émeutes préfigurant les formes modernes de la violence, apparaît. Il reste circonscrit au sein du quartier noir chez des habitants implantés de longue date et éclate à propos de commerces et immeubles détenus par des Blancs extérieurs au quartier et que les Afro-Américains pillent avant d'être tués et blessés par la police. " La motivation dans ces émeutes 
matérialistes ", note Janowitz " n'était évidemment pas due au désespoir suscité par la famine, comme c'est le cas en Inde. On est frappé par la constance des rapports relatant l'esprit de carnaval qui caractérise les premières phases de ce type d'émeutes. Ce nouveau type de débordement démarre généralement par un incident causé par la police dans le ghetto, où s'est produite la violation réelle ou supposée d'une pratique acceptée par la police. La toute première phase est généralement mauvaise et brutale ; la police est attaquée avec des jets de pierre; les foules se rassemblent et la tension monte. Ensuite, seconde phase, les vitrines sont brisées. Le contrôle social local se désagrège et la population prend conscience qu'une occasion de pillage temporaire lui est offerte. L'atmosphère change rapidement et libre cours est donné à la jubilation. Mais pour trop peu de temps. Si les foules ne sont pas dispersées et si l'ordre n'est pas restauré, la troisième phase de l'émeute intervient: incendies, plastiquages, balles de tireurs isolés déclenchant la répression de la police et des soldats en uniforme " 9 .

Les formes modernes de la violence urbaine aux Etats-Unis

11 La description précédente pourrait s'appliquer point par point (bien que le terme carnaval soit peut-être excessif pour rendre compte du caractère jubilatoire et ludique du défoulement collectif), aux émeutes raciales des années 1960, à celles de Miami en 1980 de Los Angeles et de San Francisco en 1992, mais également aux émeutes de Brixton en Grande-Bretagne au début des années 1980 et à celles des banlieues françaises au début de la décennie suivante. A la différence près que les lignes d'identification dans les mobilisations émeutières américaines révèlent toujours une forte homogénéité ethnique et territoriale et que d'autres facteurs interviennent désormais pour distinguer la violence urbaine américaine en altérer l'ampleur, la nature, les modes d'expression et les causes.

L'ampleur

C'est une idée américaine que les villes connaissent des cycles de vie et que, comme Detroit, lorsqu'elles " rouillent " dans la FrostBelt, il faut les abandonner tels les Grecs brûlant leurs vaisseaux derrière eux (les habitants de Jérusalem ou de Rome apprécieront). Dans les villes que les Américains aisés ont déserté (seuls $12 \%$ de la population vit dans les 24 métropoles de plus de 500000 habitants), le nombre d'homicides, de viols, d'attaques à main armée a plus que doublé en trente ans. Les statistiques de 1991 ont dépassé celles de 1990 avec 25000 homicides recensés par le FBI, chiffre jamais atteint, soit une moyenne de 400 homicides par semaine. Soit plus du double de la France. Selon un rapport du département de la Justice émis par le Bureau des statistiques judiciaires, les taux d'homicide pour 100000 habitants sont quatre à cinq fois plus élevés aux Etats-Unis qu'en Europe ; les viols le sont sept fois plus, les vols à main armée quatre à dix fois plus ${ }^{10}$. Quant à la petite délinquance, elle est deux fois plus répandue, elle aussi, qu'en Europe. Si, selon les statistiques officielles, un passager sur 595.000 se fait agresser dans le métro parisien, les chances de l'être sont huit fois plus élevées à New York où le métro fonctionne jour et nuit et traverse des kilomètres de quartiers dévastés ${ }^{11}$. Dans toutes les grandes villes, sur dix ans, la criminalité est en augmentation: de $56 \%$ à Boston, de $28 \%$ à Philadelphie, Chicago, Miami, Oakland. A Washington, on a recensé plus de 8000 homicides chez les Noirs en 1991 et à New York plus de 3000 viols et 68000 agressions " ${ }^{12}$. Tandis que 24 Américains étaient tués pendant la guerre du Golfe, dans le même temps 52 Américains étaient assassinés à Dallas ${ }^{13}$. En trois jours d'émeutes raciales à Los Angeles, 58 personnes ont perdu la vie, soit plus qu'à St. Louis en 1917 (48 morts), qu'à Detroit en 1967 (43 morts) et qu'à Watts 
en 1965 (34 morts). Le bilan de Los Angeles donne plus de deux mille trois cents blessés et un milliard de dollars de dommages (selon les agents d'assurances) " 14.

Noirs, 75 \% des Latinos par des Latinos, 55 \% des Blancs par des Blancs. Les Noirs aujourd'hui à New York ont plus de chance d'être tués que lorsqu'ils partaient pour le Vietnam. Le taux de victimisation est dix fois plus élevé pour les Noirs que pour les Blancs (entre 15 et 44 ans) et cinq fois supérieur pour les Noires que pour les Blanches. Ces données brutes n'expliquent pas la réalité des faits. De nombreux Américains redoutent de dénoncer à la police ce dont ils ont été victimes : selon un sondage, une famille sur trois aurait subi un acte criminel grave ${ }^{17}$.

Les modes d'expression de la violence

Hormis l'expression par l'émeute et le feu à laquelle ont recours les communautés noires et latinos pour riposter aux provocations racistes dont elles sont l'objet, celle de la violence-rupture, nouvelle stratégie d'expression lorsqu'il n'y a plus d'espoir de se faire entendre autrement qu'une arme à la main jusqu'à l'autodestruction à laquelle se livre une grande partie de la jeunesse noire contemporaine par le truchement de la drogue, la manifestation collective et individuelle de la violence est protéiforme et se nourrit d'elle-même. ville, soit de 1934 à 1955, par exemple, disent qu'alors ils ne se souciaient pas de leur sécurité personnelle, tandis qu'il existe quelque chose de spécifique, de différent et d'effrayant dans la vie urbaine américaine d'aujourd'hui ${ }^{18}$. Certes des groupes ont recours à la violence pour une cause précise (un verdict inique), pour mobiliser leurs ressources ou accroître leurs gains. Mais dans la plupart des cas, ce n'est pas en vue d'une riposte, de demandes de formes de protection ou de sanction, comme ce fut le cas à l'époque du mouvement pour les droits civils, puis du Black Power, que surgit la violence ${ }^{19}$. Elle est plutôt devenue chez les jeunes des centres villes un style de vie à base de pulsions qui autorisent à tuer pour une dent en or convoitée, pour une commande de cheeseburgers bâclée, pour un soda ${ }^{20}$. Parfois le meurtrier dit avoir tiré au hasard, parce qu'il en avait envie, mais il s'agit plutôt de la mise en action d'un des trois ressorts qui sont à l'origine de la violence : la peur devant une situation inconnue ou devant des personnes perçues comme des assaillants potentiels, le défi qu'on se lance à soi, l'envie d'améliorer son image de dur vis-à-vis des autres . ${ }^{21}$.

Gangs, Mafia et politique

Dans les quartiers à forte criminalité, les gangs qui tiennent les rues sont, dans la majorité des cas, connus des habitants, ils sont l'émanation des quartiers populaires. 
New York compte 50 gangs avec 5.000 membres (de 9 à 40 ans), Chicago, 125 avec 12500 membres; Dallas 225 gangs, mais le record est battu avec les 600 gangs de Los Angeles qui regrouperaient quelques 70000 jeunes ${ }^{22}$, ce qui expliquerait en partie le déchaînement de violence dont la ville a fait l'objet au début du mois de mai 1992. La tradition d'insertion dans un gang urbain est ancienne. A l'époque de la Frontière, les gangs pillaient les diligences et attaquaient les saloons. Avec les vagues d'immigration, ils se sont dirigés vers le désert urbain et de chaque groupe ethnique a émané des formations de gangs. La célèbre étude de $\mathrm{F}$. Trasher en dénombre 1300 à Chicago dans les années 1920. A Los Angeles, un charpentier de 47 ans, père de quatre enfants, affirme: "bien que je n'aime pas tous ces coups de feu qui font partie intégrante des gangs, je suis fier que mes fils appartiennent au gang $(\mathrm{X})$ car cela maintient la tradition familiale; mon père en faisait déjà partie. Cela est dans la tradition du quartier et ce n'est pas aussi mal que les médias veulent bien le dire. Le gang a aidé notre quartier... et quant à la violence, eh bien, je ne m'en fais pas parce que, c est comme s'ils étaient dans l'armée, il se pourrait qu'ils soient tués, mais ça tient à la Providence car avec la vie, va la chance ${ }^{23}$. Rationalisation, résignation? Ce témoignage entérine l'image de valeureux guerriers que se donnent les gangs. Pour certains, les gangs sont la seule expérience affective qu'ils aient jamais eue. En faire partie correspondrait dans d'autres cultures à l'engagement chez les scouts ou aux jeunesses communistes.

Pour qui ne les connaît que par le truchement des médias, il est impossible de comprendre l'essence des actes de violence perpétrés par les gangs et l'absence de remords après les forfaits les plus violents. Or dans ces quartiers populaires, la violence est un état naturel et nécessaire à la survie. Elle sert à maintenir la crédibilité de l'individu, à le protéger des exactions perpétrées par ses clients, sa bande, ses voisins. Elle doit être systématique et répétitive de manière à établir une réputation. La violence est monnaie courante dans un monde hobbesien où les habitants sont en état de guérilla permanente pour s'approprier les moindres ressources. Telles des mafias, les gangs exercent un contrôle territorial sur des zones que l'appareil d'Etat ne cherche plus à contrôler.

La violence est d'autant plus dure à supporter qu'une partie de la population exerce un travail tout en habitant dans un quartier où règne une forte insécurité. Mais pour le gang, la violence est la monnaie de son économie, avec toutefois des contraintes imposées dans son usage, de peur de représailles, de souffrances, et en raison d'un code de conduite assez vague faisant référence au respect et à l'honneur. Des rituels guerriers pour exorciser la peur donnent aux membres des gangs le sentiment de leur invulnérabilité et de leur immortalité. Interviewés, les jeunes membres des gangs évoquent à tout propos ceux des leurs qui sont "tombés" pour le gang dix ou vingt ans plus tôt, constituant de ce fait une histoire orale mythique qui anesthésie la peur de la mort et ses représentations. L'action des gangs commence par des mesures routinières de représailles contre des gangs voisins appartenant à d'autres ethnies, Italiens, Irlandais, Africains-Américains, Coréens, Chicanos détenteurs de territoires et des ressources convoitées. Si la répression tarde à venir, si le pouvoir "flotte" comme dans le cas de Los Angeles, leur rituel de violence dépasse la limite des quartiers minoritaires, elle prend un tour symbolique, de portée "thérapeutique", visant la destruction des propriétés matérielles voire des vies des Blancs, témoins d'un rêve inaccessible. "Quand on se sent frustré, l'avantage de se défouler dans un autre quartier, c'est qu'on s'éclate sans peur de représailles à quoi cela servirait de changer sa frustration en anxiété ?" s'exclame Kite, membre d'un gang jamaïcain à New York. 
"J'ai défoncé le pare-brise de la Jaguar de ce Blanc, de ce fils à papa. J'ai lancé une brique dessus parce que je hais ces enculés. Ils ont tout parce qu'ils sont blancs et que leurs pères se font $\mathrm{du}$ fric en vampirisant les autres. Je vais vous dire, cela me fait $\mathrm{du}$ bien de leur en faire baver un peu. (Portoricain, New York)" ${ }^{24}$.

Les nouvelles causes de la violence

21 Criminalité, gangs, drogue et armes à feu sont liés. Les guerres de gangs ont fréquemment pour objet le contrôle du trafic de stupéfiants. Selon l'enquête sur la toxicomanie réalisée par le Département de la santé et des ressources humaines (HHS), les Etats-Unis consomment $60 \%$ de la production mondiale de drogue, soit 13 millions de foyers toxicomanes en 1990 (on en dénombre 150000 en France), 4,5 millions de cocaïnomanes et 750000 héroïnomanes ${ }^{25}$. Non seulement les Etats-Unis consomment, mais ils sont devenus les seconds producteurs de marijuana. Il y a donc là un marché lucratif que le syndicat du crime n'est plus seul à contrôler. De plus en plus de jeunes s'adonnent à ce commerce, parce que l'économie de la drogue est le seul employeur et le seul pourvoyeur de ressources dans ces quartiers abandonnés des investisseurs et des institutions ${ }^{26}$. Prenons l'exemple de Milwaukee. Entre 1979 et 1986, 50000 emplois ont été supprimés dans l'industrie, et les premiers touchés ont été les Afro-Américains : ils travaillaient à $40 \%$ dans le secteur secondaire en 1980, dans les ghettos noirs ils ne sont plus qu'un quart à avoir ce type de travail en 1989. Près de la moitié d'entre eux et les deux tiers de leurs enfants sont désormais dépendants de l'assistance publique (welfare). Dans ces conditions, la coupure naturelle avec le gang qui s'opérait vers l'âge de vingt ans, âge d'entrée dans la vie active ne se fait plus, les plus âgés restent là, s'impliquent dans les activités illégales et y entraînent les plus jeunes. Leur modèle n'est ni Jesse Jackson ni Martin Luther King mais Al Capone. Le gang devient dysfonctionnel pour le quartier. Les plus jeunes, grâce à l'argent de la drogue, forment les nouveaux chefs de famille. La drogue leur procure à la fois des revenus et des raisons de vivre. Comme le note le sociologue Ph. Bourgois, ils partagent une idéologie, des valeurs et des symboles qui sont partie intégrante d'une culture de rue exclue du reste de la société américaine et de son économie mais qui en sont aussi l'émanation. Ces parias, en fait, n'acceptent pas d'être relégués au fond d'une ornière. Ils utilisent tous les moyens, tout comme les chefs d'industrie au tournant du siècle et comme les Golden Boys de Wall Street aujourd'hui pour gagner rapidement de l'argent, se donner une image de durs, structurer leur vie. Mais, c est ce processus même contre et à l'intérieur du système qui exacerbe le traumatisme de leur communauté et détruit annuellement par la violence qu'il engendre des centaines de vie à l'échelon individuel.

Les gangs sont armés. L'accès des gangs aux armes les plus sophistiquées est sans aucun doute la cause principale du taux d'homicide élevé des Etats-Unis. La dernière livraison des fabricants de Floride propose des armes particulièrement légères pour les enfants et imperméables aux empreintes digitales ${ }^{27}$. La vente des armes militaires semiautomatiques d'attaque a décuplé en deux ans, après les émeutes de Los Angeles, les armuriers de la ville en ont vendu quatre fois plus qu'à l'accoutumée. Il y a aujourd'hui presqu' autant d'armes que d'adultes aux Etats-Unis. Or, $60 \%$ des meurtres se font par armes à feu. La technologie facilite l'attrait psychologique des jeunes pour les armes. Biggie, Chicano de 16 ans s'en explique : "Je me souviens quand le gang m'a demandé pour la première fois de descendre quelqu'un. J'étais tendu, mais j'avais ce pistolet automatique et quand j'ai commencé à tirer, mon vieux, c'est devenu facile. C'est pour ça que c'est si facile, c'est rapide et il $\mathrm{n}$ y a rien de personnel comme quand on utilise un couteau. Eh, tu sais ce qui me plaît, quand je porte ce pistolet, c'est qu'on te respecte 
qui que tu sois ou quelle que soit ta taille (Biggie fait 1,60 m), cela remet tout à niveau" ${ }^{28}$.

Certes, dans la vie quotidienne, la fonction des gangs est d'assurer la défense du territoire, de se poster aux coins des rues, de repérer tout mouvement suspect; ils agissent comme des "pairs vigiles" contre les prédateurs, les spéculateurs, les parasites de leur quartier. Mais inversement, des débordements pathologiques peuvent se produire, tenant à la guerre des gangs. En 1988, à Los Angeles, les Crips et les Bloods (145 sections) se sont mis à tirer de leur voiture au hasard comme pour signifier aux habitants de certains quartiers que leurs gangs étaient inaptes à les protéger. Les habitants n'ont pas tardé à faire exceptionnellement appel à la police, tant le niveau de tension intra-communautaire entre les familles pauvres détentrices d'emploi et les gangs de l'underclass était devenu intolérable. Sans appui des institutions communautaires de quartier, églises, travailleurs sociaux qui se trouvent désormais à la périphérie des ghettos, les habitants n'avaient d'autres ressources que de s'armer, appeler la police ou déménager. Cette guerre, en effet, avait causé des centaines de morts et de blessés. Mais les opérations de police à large envergure ("sweep") sont rares, car la police se sert des gangs pour obtenir un certain nombre d'informations sur la vie de la rue et les gangs ne sont pas sans coopérer à la corruption de certains membres de la police. D'où un ajustement mutuel, des "transactions collusoires", selon l'expression de M. Dobry, plutôt qu'un véritable contrôle.

Une société atomisée en communalismes possessifs

Des facteurs plus profonds que l'existence des gangs, la vente libre des armes, la drogue et l'argent rapide qu'elles procurent peuvent expliquer la nature différente de la violence dans la société américaine.

On peut d'abord s'interroger sur l'impact des très grandes métropoles américaines, porteuses d'éléments pathogènes. Par rapport à l'environnement urbain européen plus inclusif, quoi qu'on en dise, le tissu social américain est beaucoup plus distendu; géographiquement, l'agglomération de Los Angeles s'étend sur une centaine de kilomètres, par exemple, et la ville n'est pas tenue. Plus de deux cents juridictions sont responsables de services municipaux et lèvent leurs propres impôts. Pour le gouverneur républicain, la "dysharmonie" que présente la ville de Los Angeles n'apparaît pas comme une priorité. Dans de telles mégalopoles, le marquage ethnique territorial de quartiers et de résidences isolés les uns des autres est assorti d'une forte segmentation de classes et d'un "communalisme possessif" qui amène à tirer à vue sur ceux qui ne sont pas connus ou conformes ${ }^{29}$. L'hostilité mutuelle, l'indifférence des groupes raciaux et ethniques sont intensifiées par la forme rigidement ségréguée d'agglomérations urbaines très diversifiées d'où s'est évanoui le sens civique d'une démocratie urbaine où chacun trouverait sa place. Au contraire, la majorité des confrontations ethniques territoriales a pour origine une perception $\mathrm{d}^{\prime}$ 'invasion" qui se solde par des coups de fusil tirés par des vigiles ou des voisins "renifleurs" (nosy neighbours) fiers de leur fonction légitime de guetteurs et opérant fréquemment en association avec la police locale. Ce self help qui, en France, serait interprété comme de la délation ou comme une dangereuse dérive de la société civile amenée à se faire justice elle-même est au contraire encouragé tant par les institutions que par les citoyens et permet de comprendre la popularité du port d'armes.

Un multiculturalisme exacerbé 

inquiétante de la société américaine: du pluralisme dominé par un centre hégémonique jusqu'aux années 1970, on en est arrivé progressivement sous l'impulsion des tribunaux sollicités par des minorités en mal de justice à l'excès d'un multiculturalisme parcellisé où chacun excipe de ses droits avec la plus grande intolérance sans se soucier de l'intérêt général. La disparition de la religion "civile" qui avait certes le défaut de refléter une culture blanche mâle anglo-saxonne mais le mérite grâce à des valeurs pluralistes de donner aux différentes composantes de la société des références communes met en péril le unum au profit de l'ex-pluribus; la segmentation actuelle de la société exacerbe l'auto-enfermement des individus dans leur groupe de référence. Comme le remarque ironiquement J. Chapin, les seuls qui vivent encore l'éthique de la compétence et de la discipline sont les immigrants du tiers-monde, mais ils la perdent à la quatrième génération. Il est possible, suggère-t-il, que l'Amérique ait vécu de la vitalité immigrée et qu'elle fasse à présent l'expérience de la désagrégation, "problème de la quatrième génération ${ }^{30}$. Le problème de l'inner-city vient de ce que non seulement les autres groupes s'en soucient peu (les Coréens, par exemple, qui tiennent les boutiques dans les quartiers noirs vivent entre eux dans d'autres quartiers) mais qú ils sont incapables, comme le montre le verdict de Simi Valley, de se représenter ce que peut être la vie de jeunes Chicanos, Vietnamiens ou Afro-Américains dans le quartier de South Central de Los Angeles. On ne peut nier que la forme urbaine et démesurée dans laquelle évoluent les groupes sociaux sustente ce déchaînement meurtrier, plus que ne l'ont fait les communautés rurales et décentralisées des siècles précédents, encore que la violence symbolique et morale y ait été d'une grande emprise.

De nos jours, une grande partie de la violence américaine est de caractère urbain, mutilant et automutilant et par conséquent anxiogène pour la majorité des Américains. Dès 1919, l'ethnologue Robert Luke Jenny, de manière prémonitoire, associait violence et forme urbaine. Une charge émotionnelle considérable ne peut que naître du développement urbain sélectif de l'Amérique moderne, annonçait-il. "L'absence d'harmonie urbaine aboutira fatalement au rejet par certains des solutions imposées par les autres, rejet qui pourrait prendre la forme d'un suicide collectif adopté par les sociétés primitives en péril ${ }^{31}$.

Aucune courroie de transmission objective du mécontentement, des modes de pensée de ses habitants-là, ne parvient jusqu'aux autres, ne serait-ce que parce que la définition stigmatisante de l'extrême pauvreté aux Etats-Unis s'attache aux comportements déviants de l'underclass (blâmer la victime) et n'établit pas de lien entre conjoncture économique et pauvreté. Aussi, contrairement à ce qui se passe en Europe, où une définition plus large de la pauvreté relative fait une large part au chômage, aux choix gouvernementaux et aux pratiques des institutions financières, les Américains aisés ne se sentent pas responsables des habitants du ghetto et la corrélation entre l'enrichissement des plus riches (le $1 \%$ d'entre eux qui détient plus en richesse que les $90 \%$ restant a augmenté ses gains de $102 \%$ après impôts entre 1977 et 1989) et l'appauvrissement des revenus des plus pauvres (de $10 \%$ pendant la même période) n'est pas établie ${ }^{32}$.

Un État-méduse

La dérive des institutions tentaculaires et faibles de l'Etat envahissant et prisonnier des intérêts particuliers, a été accentuée par l'esprit de dérégulation reaganien. L'Etat s'est 
désintéressé du sort des villes (l'aide fédérale réservée aux budgets sociaux locaux a été amputée des deux tiers depuis 1980 et représente moins de 15 milliards de dollars) et a passé cyniquement le fardeau aux Etats et aux villes endettées et limitées dans leurs dépenses par la volonté des contribuables. Les plus pauvres ont subi de plein fouet cette politique et en ont tiré les conséquences. C'est comme si on avait pris à contre-pied la remarque de bon sens d'un membre d'un gang à qui l'on demandait comment il résoudrait le problème posé par l'existence des gangs si le gouverneur lui offrait un million de dollars. La réponse avait été claire : le plein emploi. Or, au cours des dix dernières années, lorsque les emplois ont quitté les centres villes pour des sites plus lucratifs, aux plus hauts sommets les autorités sont restées passives.

L'urbain est un terme qui a disparu du vocabulaire de Washington(34). En voulant les délivrer de "la toile d'araignée de la dépendance", R. Reagan a conduit les plus pauvres des pauvres (on estime l'underclass à un million de personnes environ sur une pauvreté touchant entre 33 et 50 millions de personnes) à un "état de dépacification" sociale, selon l'expression de N. Elias c'est-à-dire à une situation de luttes, de larcins, et d'activités illégales à des fins de survie. La pauvreté, on le sait, favorise la violence par l'état de crise qu'elle engendre. La société américaine récompense fortement ceux qui réussissent et rejette les autres. Ces "autres", non "méritants", ont bien peu à perdre lorsqu'ils pillent, incendient, et mettent à sac une ville. Discriminés par la couleur de leur peau et par leur pauvreté, ils ne se sentent pas liés par un pacte à l'Amérique blanche et leur ségrégation territoriale dans des quartiers fort étendus (le ghetto sud de Chicago a la taille de Paris et de sa périphérie) les dissuade d'en sortir, alors qu'en quelques stations de RER, les jeunes des banlieues parisiennes se trouvent au coeur de Paris). A cela, il faut ajouter le poids d'une culture de rue qui n'incite pas les jeunes exclus à "réussir" à la manière des Blancs. Aller à l'école, trouver un travail, c'est imiter les Blancs. Aussi peut-on comprendre que lorsque l'occasion leur en est donnée, l'action violente serve de moyen de communication pour asseoir leur position dans les débats de l'heure. La violence et la destruction symbolisent aux yeux du monde tout ce que leur situation a d'immonde et de désespéré ainsi que toute l'étendue et la profondeur de leur colère. La violence est pensée comme un message adressé à la communauté blanche environnante ("terrorisme de contact" dirait Elias), une menace qui annonce que les conditions intolérables faites aux Noirs ne seront bientôt plus supportées. L'extrême sélectivité des cibles choisies pour être détruites prouve, s'il en était besoin, la prédominance du côté symbolique sur le rôle expressif, également présent, il est vrai. Lorsqu'un conflit précis se déclenche dans un système déjà en crise et a fortiori lorsqu'il y a une écoute (période électorale et quotient de sympathie dans la population émue par l'injustice du verdict), il active l'expression de la violence. La rébellion sociale se conjugue alors à une rébellion de nature politique et culturelle ${ }^{33}$.

Paradoxalement, l'incapacité de l'Etat américain à prendre en charge le problème posé par les quartiers pauvres (l'absence de discussion au Congrès de ce problème qui ne touche que des abstentionnistes) se dote d'une grande difficulté à contrôler les Etats dans l'Etat, c'est-à-dire non seulement à réduire l'excès d'autonomie contre-productif des échelons sub-nationaux mais à intervenir sinon directement du moins par le biais des commissions concernées lors d'abus flagrants. Imagine-t-on un seul instant en France que si, depuis quatorze ans, dans la seconde ville du pays, des centaines de plaintes pour brutalité et corruption émanaient régulièrement de la part de citoyens de toutes origines, aucune mesure ne serait prise à un échelon supérieur? A Los Angeles, le maire, de par la volonté du corps électoral, a aucun pouvoir sur le chef de police son 
rival. Des membres des gangs de Los Angeles rapportent que la police les a fréquemment roués de coups, qu'ils ont assisté aux meurtres des leurs dans les conditions les plus lâches. La conversation échangée entre policiers lors du tabassage de R. King incite fortement à les croire. La police de Los Angeles ne se comporte pas mieux que les gangs auxquels elle faisait la chasse. La volonté de vengeance entretenue par les jeunes qui ont subi humiliation et violence policière ne peut qu'être source d'autres violences ${ }^{34}$. On sous-estime souvent cet élément dans l'analyse des émeutes : le système policier considère le ghetto comme un territoire ennemi où l'on doit "tirer pour tuer", selon l'ordre donné à sa police par le maire de Chicago, R. Daley Crime organisé et activités de gangs sont autant d'écosystèmes parallèles mis en place pour affronter cette violence policière. Dans certaines villes, les leaders noirs ont demandé aux majorités politiques tenant les municipalités ou les comtés de leur donner une police moins "raciste". Lorsqu'ils n'ont pas été écoutés comme à Miami, la brutalité des policiers sudistes ou cubains a provoqué plus de quatre fois en dix ans les déchaînements de la foule. En revanche, on peut citer l'action du maire de New York, D. Dinkins, qui depuis 1991 quadrille les quartiers durs avec des îlotiers, s'appuie sur des volontaires et a mis en place un maillage institutionnel d'encadrement qui a porté ses fruits lors des émeutes de 1992. Il y a d'autant lieu de la signaler que la politique de prévention est excessivement rare aux Etats-Unis.

La violence comme culture

Pour terminer, on ne peut ignorer de surcroît que les représentations caractéristiques de l'imaginaire américain donnent une place prépondérante à la violence". Pour ne prendre qu'un seul exemple, la télévision comme le cinéma donnent du syndicat du crime organisé, la Mafia, une image romantique et pernicieuse. Organisée sur des structures familiales, la Mafia apparaît comme une entreprise très lucrative, surpassant les 500 premières entreprises dont la liste apparaît annuellement dans le magazine Fortune. Les signes de consommation ostentatoire des membres de la "famille" correspondent aux symboles de la réussite dans la société américaine. Mais, par opposition aux autres Américains que la réussite éloigne de leur communauté d'origine, les membres de "la famille" restent étroitement soudés et attachés à leur quartier et à ses valeurs d'origine. Les Mafiosi les plus célèbres tels Lucky Luciano ou Frank Costello, partis du bas de l'échelle, donnent l'impression dans les récits qui les célèbrent en tant que self made men, d'avoir atteint les sommets grâce à leur compétence innée ainsi qu'aux risques et à l'initiative qu'ils ont pris aux moments opportuns. William Simon, ancien membre du cabinet de R. Reagan, répète, à quelques variantes près, la même idéologie: "l'expérience montre que Dieu a donné une abondance de compétences économiques aux pauvres et que les pauvres peuvent massivement et à très court terme sortir de la pauvreté, en utilisant les talents que Dieu leur a donnés". Comme le note le chercheur sicilien-américain, E. Li Puma, les représentations populaires de la Mafia occultent la réalité de la violence et de la corruption dans la société américaine. En mettant l'accent sur la réussite financière de cette société parallèle et sur l'importance de la solidarité familiale, elles contribuent à masquer les liens intrinsèques de la société américaine et du capitalisme et à promouvoir à la fois l'idée que la chance et la mobilité résultent de la seule initiative individuelle (et non des facteurs structurels de classe et de race) et que la famille est indépendante de la dynamique de l'économie politique dans laquelle elle s'insère ${ }^{35}$.

Des conflits circonscrits 

manifestée les Etats-Unis, ce n'est pas la prévention mais la répression, l'incarcération et l'exécution capitale. Le taux d'incarcération américain est le plus fort du monde, devant l'Afrique du Sud, avec 1,2 million de prisonniers au coût de $40000 \$$ annuels. (Par comparaison, le coût des études dans une université d'Etat est environ de 6000 \$ et de 20000 \$ dans une université prestigieuse). Ce taux avait doublé en 1980 et il a encore doublé en $1990^{37}$. Après les émeutes de Los Angeles, 17000 personnes ont été arrêtées dont une majorité de Latinos; certains membres des gangs sont venus se rendre spontanément. Même si le surpeuplement carcéral, l'ampleur de la récidive (dans plus de $70 \%$ des cas) et la force du contre-modèle de la culture de la rue qui donne du prestige à quiconque a tâté du pénitencier font douter de l'efficacité de cette politique pourtant populaire, les données révèlent qu'il y a aujourd'hui 609600 jeunes afroaméricains "neutralisés" pour un temps dans les prisons (contre 436.000 sur les campus). 36 Etats admettent la peine de mort (dont 28 pour les prévenus mineurs et les 
débiles mentaux). Les $3 / 4$ des exécutions ont lieu dans les Etats du sud: Texas, Floride, Louisiane et Géorgie. On sait qu'il y a plus de risques à encourir cette peine pour les hommes, les Noirs et les individus issus des minorités ${ }^{38}$. En conclusion, le bilan comparatif indique que si les processus de logiques émeutières sont identiques à Vauxen-Velin, Brixton et Los Angeles, le contexte historique, politique, économique des Etats-Unis offre une clé d'explication distincte : la soif et la capacité d'enrichissement individuel sans précédent au cours des années 1980, la croissance de l'abîme séparant les nantis de ceux qui sont privés du capital économique, social et culturel qui leur permettrait de rejoindre les premiers, l'absence de régulation et de propositions constructives d'un Etat endetté et conservateur, la disparition progressive des valeurs humanistes et intégratrices annoncent pour les années à venir une spirale de la violence, violence protéiforme et essentiellement urbaine. L'Europe aujourd'hui comme par le passé n'est pas à l'abri de grands désordres, mais la segmentation moins accusée des populations (tant en matière de richesses que d'origines ethniques), les transferts opérés par les Etats en faveur des plus précarisés et, il faut le dire, la plus forte intégration idéologique des sociétés européennes ne l'aligne pas à ce jour sur le modèle américain de violence urbaine.

\section{NOTES}

2. Graham H. D., The paradox of American Violence, p. 279 in Graham H. D. et Gurr T., Violence in America, Beverly Hills : Sage, 1979, 2e ed., 528 p.

3. On doit cette expression à Higham J., Strangera in the Land: Patterns of America Nativism, 1896-1925, New Brunswich, N. J. : Rutgers Univ. Press, 1955.

4. L1intransigeance des colons puritains à l1égard des dissidents tels Roger Williams conduisait ceux-ci à s1exiler et à fonder leur colonie ailleurs (ici Rhode Island). La mobilité a fréquemment été la méthode adoptée par un individu ou un groupe pour résoudre des problèmes sociaux.

5. Le darwinisme social connaît un grand succès à la fin du XIXème siècle. Dans les années 20 , les sociologues de l1École de Chicago, étudiant les phénomènes urbains, montrent que les groupes ethniques se déplacent sur l1espace urbain selon un processus en trois temps : invasion de l1espace occupé par un groupe, résistance temporaire de celui-ci, succession des nouveaux arrivants. Cette théorie de la succession connait des variantes et s1applique mal aux Noirs. S. Body-Gendrot (avec C. Lefèvre et al), Les villes des Etats-Unis, Paris, Masson, 1987.

6. Bass Warner S., Thc Private City : Philadelphia in Three Periods of its Growth, Philadelphia Temple, 1968 ; cette thèse est corroborée par R. Lane, " Urbanization and Criminal Violence in the 19th Century : Massachusetts as a Test case " in H D. Graham et $\mathrm{T}$ Gurr (eds.), Violence in America : Historical and Comparative Perspectives, New York, Praeger 1969, ch. 12.

7. Lane R. Roots of Violence in Black Philadelphia, 1860-1900, Cambridge, Mass. : Havard Univ. Press, 1986 ; Danchin S., " Etats-Unis : deux siècles d1émeutes ", Libération, 8/6/1992. 
8. Janowitz M., " Collcctive Racial Violence ", p. 264 in H. D. Graham et T Gurr, op. cit. 9. Janowitz M., op. cit. p. 267 ; Keith M., " Que s1est-il passé ? Les émeutes de 1980 et 1981 dans les cités britanniques. Un essai d1analyse ", Revue européenne des migrations internationales, vol. 6, n 31990 ; Farid, Voilà pourquoi la haine ! ", Les Temps modernes, $n^{\circ}$ 545-46, déc. 1991, p. 104.

10. Rapport de la Fondation Eisenhower, "Doing what works", juin 1990.

11. S. Body-Gendrot, "Réalité et imaginaire de l1insécurité", Projet n 194, juillet-août 1985, pp. 19-31.

12. International Herald Tribune, $1 / 8 / 91$; Libération, $20 / 1 / 92$.

13. "1990 : The Bloodiest Year Yet ?", Newsweek, 16 juillet 1990.

14. Le Monde, 6 mai 1992.

15. National School Safety Center, News journal, printemps 1991, p. 20. 15. Children's Defense Flind, Childrcn 1990 : a Report Card.

16. Children's Defense Fund, Children 1990 : a Report Card.

17. "All about Crime", New York, 3 sept. 1990.

18. Témoignage de l'historien $M$. Dubosky à l'auteur.

19. Sur la violence instrumentale du mouvement pour les droits civiques et du Black Power, Body-Gendrot S., Maslow-Armand L., Stewart D., Les Noirs américains aujourd'hui, Paris, A. Colin, 1984.

20. Titres repris du New York Times et du Washington Post. Cf. The Economist, $10 / 8 / 91$.

21. Selon l'International Herald Tribune (24/7/86), seuls $25 \%$ des crimes sont fortuits et commis par des inconnus au hasard dans la grande ville.

22. National School Safety Center Newsjournal, nov. 1991, p. 1 ; selon la police, il y aurait 250 jeunes dans les gangs de Los Angeles, Le Monde, 8/9/88.

23. Sanchez Jankowski M., Islands in the street, Berkeley : Univ. of California Press, 1991, p. 181.

24. Sanchez Jankowski M., op. cit. p. 156, 159.

25. Rapport de l'ONU cité dans le Monde, 14/1/92 ; "Heroin : a different battle", International Herald Tribunc, 11/2/92.

26. "In cities, Poor Families are dying of crack", New York Times, 11/8/89. Voir également T. Williams, Cocaine Kids, Flammarion, 1990.

27. International Herald Tribune, 13/3/92.

28. Sanchez Jankowski M., op. cit, p. 172.

29. Plothin S., "Community and Alienation : Enclave Consciousness and Urban Social Movements", Comparative Urbnn and Community Resenrch, vod. 3, 1991 ; BodyGendrot S., "La ségrégation aux États-Unis", Cahiers de l'Habitat, vol. 15, oct. 1991. 30. Chaplin J., "Symptômes de déclin ?", p. 44, Le Débat, mars-avril 1992.

31. Cité par Danchin S., op. cit.

32. "Renganites Rally Round the Trickle-Down Flag", International Herald Tribune, 20/6/92.

33. Freyberg J. W, "la violence, une plénitude de sens" (traduit par J. Lautman), sociologie du travail, 4 octobre 1971.

34. La charte devrait être modifiée par référendum d la suite des émeutes.

35. Li Purna E., "Interpreting the Mafia Mystique", Ethnic Groups, hiver 1982.

36. Freyberg J. W, op. cit.40. Le comportement différent en matière de solidarité des Portoricains et des Juifs orthodoxes en cas de conflit a été analysé par l'auteur dans 
"Luttes de classes et luttes ethniques dans le Lower East Side à Manhantan", Revue française d'Etudes politiques, hiver 1982.

37. Le Monde, 28/2/90.

38. Département de la Justice, bureau des statistiques, sept. 1991. Sur 2356 condamnés à mort en 1990, 40 \% sont de race noire.

\section{RÉSUMÉS}

Aux États-Unis, la violence est liée à la construction de la nation américaine et à sa culture. Cependant la violence était par le passé instrumentalisée et, mis à part peut-être pour les émeutes raciales, de nombreuses mesures ont été fréquemment arrêtées pour y mettre fin. Aujourd'hui la violence urbaine, en constante augmentation, exprime un désespoir, une aliénation et prend la forme en définitive d'une autodestruction qui semble défier toute possibilité de contrôle. Le trafic de drogue, la libre circulation des armes sont en augmentation dans des territoires véritablement abandonnés par les autorités américaines et le reste de la société. De plus en plus de gens vivent dans ces enclaves, marquées et caractérisées par l'existence au sein de la population d'un fort sentiment d'appartenance communautaire, et où la démocratie urbaine est en chute libre. La violence est ainsi le dernier mode d'expression, le dernier recours adressé par ces affranchis au reste de l'Amérique. En dépit de similitudes avec les processus qui ont conduit aux émeutes en Europe, le contexte socio-économique actuel des ÉtatsUnis fait toutefois de ce pays un cas à part.

In the United States, violence is linked to the creation of the nation and to its culture. In the past this violence was purposeful and, perhaps with the exception of race riots, usually controlled. Today's urban violence is rising and expresses despair, alienation and self-destruction to an extent that seems uncontrollable. Drug and arms traffic are constantly increasing in zones more or less abandoned by authorities and by society in general. The population of these zones is growing, characterised by a strong feeling of forming a community apart, and urban democracy has virtually disappeared. Thus violence is the ultimate message sent by these " emancipated " groups to the rest of America. Although there are numerous resemblances with certain situations in Europe, the present socio-economic context makes the United States a case apart.

\section{INDEX}

Mots-clés : violence, villes/ sociologie urbaine

Index géographique : Etats-Unis

\section{AUTEUR}

\section{SOPHIE BODY-GENDROT}

Politiste, professeur de civilisation américaine à la Sorbonne et rédactrice en chef de la Revue Française d'Etudes Américaines. 